\title{
Allergic Reactions in Two Academic Medical Centers
}

J Gen Intern Med 36(6):1814-7

DOI: $10.1007 / \mathrm{s} 11606-020-06190-6$

(c) Society of General Internal Medicine 2020

\section{INTRODUCTION}

Although healthcare-related allergic reactions to foods, drugs, and other culprits are increasing, at least a fifth of allergies are inaccurately documented or interpreted. ${ }^{1}$ Clinicians do not routinely recognize or appropriately treat allergic reactions; even life-threatening anaphylaxis is often treated with antihistamines or corticosteroids rather than recommended intramuscular epinephrine. ${ }^{2}$

Improving allergic reaction recognition, management, and documentation requires improved detection and system-level targeting. We previously identified healthcare system allergic reactions using specialist-derived keyword search on safety reporting data ${ }^{3}$ and natural language processing on clinical notes $^{4,5}$, each followed by manual review. However, as such methods proved too time- and labor-intensive for large-scale allergy safety monitoring, we used a machine learning model to describe the epidemiology of allergic reactions at two academic medical centers (AMCs).

\section{METHODS}

Using a machine learning model trained on the free-text of 9107 manually labeled safety reports (rL, Toronto, Canada) (average area under the receiver operating characteristic curve $0.979,95 \%$ confidence interval $0.973-0.985),{ }^{6}$ we sorted voluntarily filed reports from July 1, 2008, to June 30, 2018, at two United States AMCs by their model-predicted probability of describing an allergic reaction in descending order. Reports were manually reviewed until the last 200 reports contained just one allergic event (i.e., false negative rate of $0.5 \%$ ). Data were obtained from $\mathrm{rL}$ databases; culprit allergens were manually reviewed and grouped. Descriptive statistics were reported.

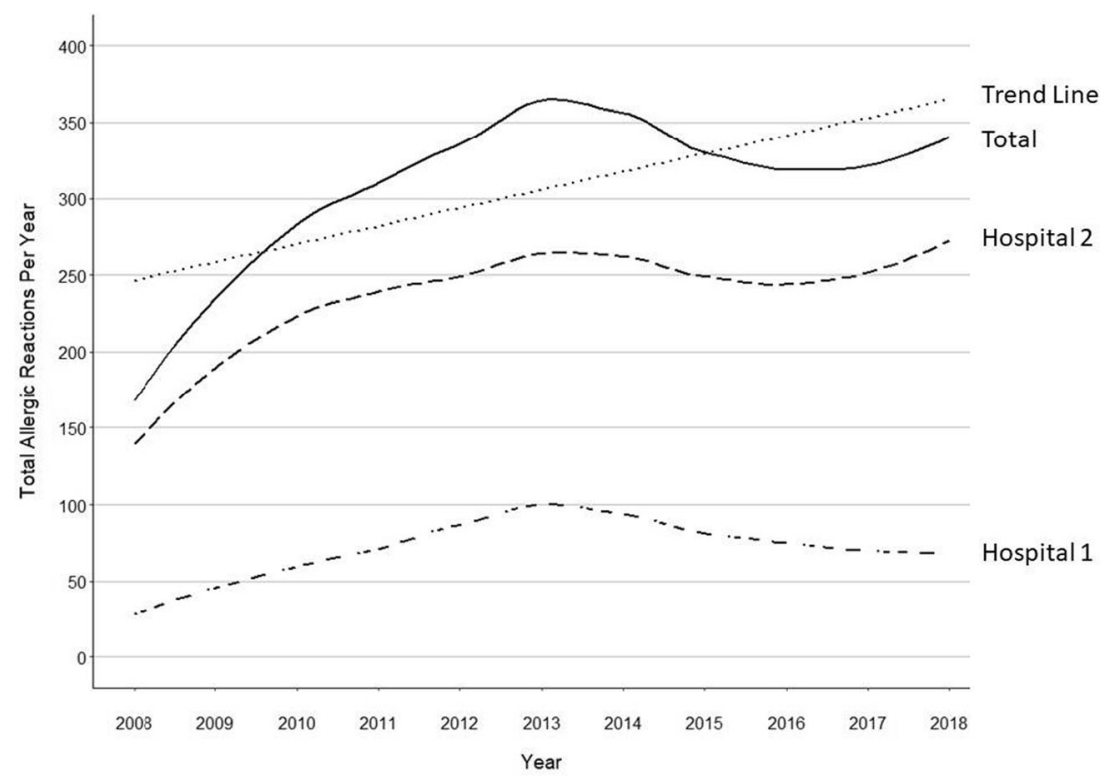

Figure 1 A decade of allergic reactions in two United States (US) academic medical centers. There were 290 (SD 75, range 141-385) mean allergy events identified per year across both hospitals. Allergic reactions increased over a decade of measurement at a rate of 11.8 reactions per year $(p<0.05)$.

Received March 19, 2020

Revised June 12, 2020

Accepted August 27, 2020

Published online September 21, 2020 

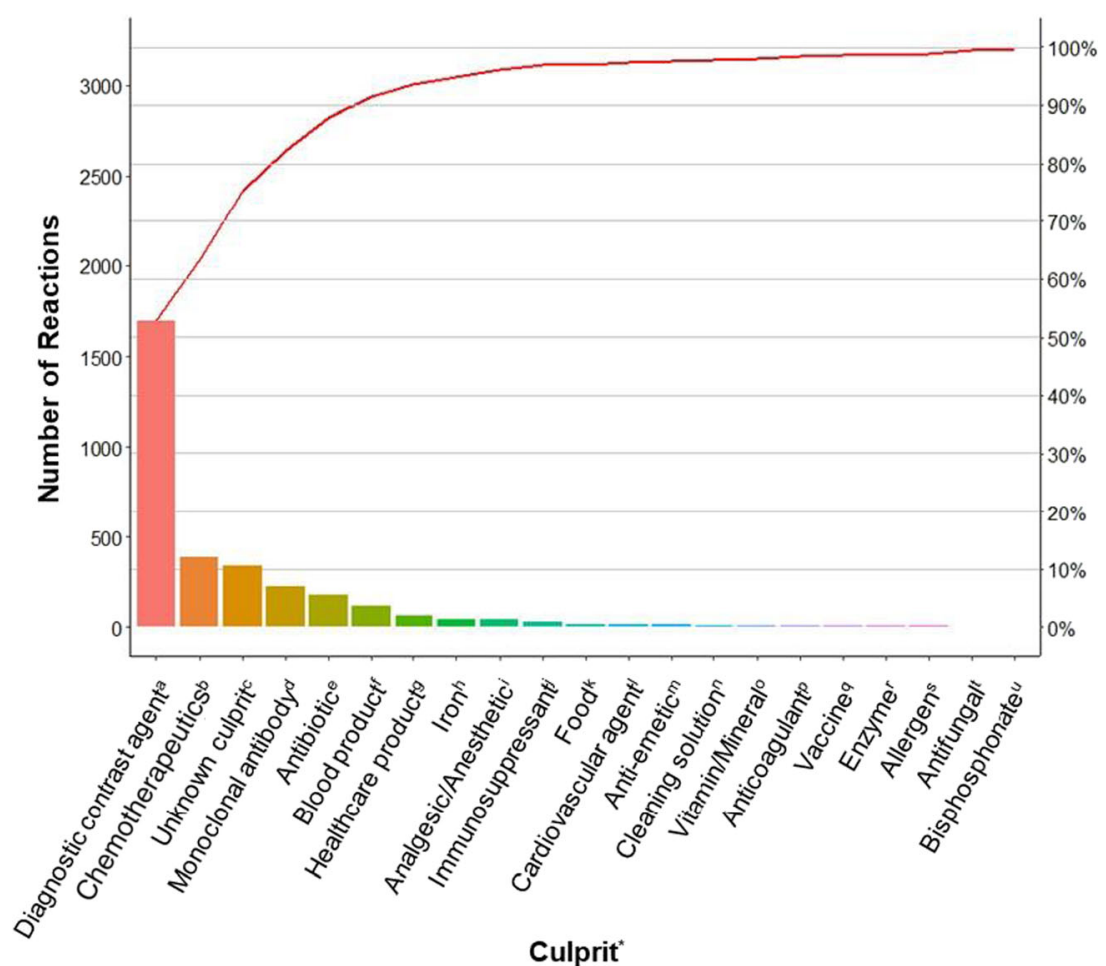

Figure 2 Allergic reaction culprits. This Pareto chart demonstrates the most common allergen culprits: diagnostic contrast agents $(n=1694)$, chemotherapeutics $(n=385)$, and monoclonal antibodies $(n=224)$. Some culprit agents $(n=164)$ were unknown. "Diagnostic contrast agent" (superscript "a") includes computed tomography contrast $(n=1081)$, magnetic resonance imaging contrast $(n=367)$, unknown contrast $(n=$ 241), ultrasound contrast $(n=2)$, fluorescein $(n=1)$, I-123 NaI capsule $(n=1)$, and nuclear medicine contrast $(n=1)$. "Chemotherapeutics" (superscript "b") includes paclitaxel $(n=117)$, docetaxel $(n=81)$, oxaliplatin $(n=64)$, carboplatin $(n=58)$, unknown chemotherapy $(n=30)$, cisplatin $(n=9)$, doxorubicin $(n=7)$, etoposide $(n=5)$, gemcitabine $(n=3)$, STA-9090 $(n=2)$, irinotecan $(n=2)$, cytosine arabinoside $(n=1)$, denileukin diftitox $(n=1)$, erlotinib $(n=1)$, imatinib $(n=1)$, pemetrexed $(n=1)$, topotecan $(n=1)$, and trametinib $(n=1)$. "Unknown culprit" (superscript "c") includes unknown culprit $(n=164)$ and unknown drug culprit $(n=103)$. "Monoclonal antibody" (superscript "d") includes rituximab $(n=105)$, infliximab $(n=58)$, natalizumab $(n=16)$, cetuximab $(n=8)$, trastuzumab $(n=7)$, nivolumab $(n=6)$, alemtuzumab $(n=2)$, brentuximab $(n=2)$, daratumumab $(n=2)$, ocrelizimab $(n=2)$, ofatumumab $(n=2)$, omalizumab $(n=2)$, pertuzumab $(n=2)$, tocilizumab $(n=$

2), ustekinumab $(n=2)$, atezolizumab $(n=1)$, daclizumab $(n=1)$, dalatizumab $(n=1)$, ipilimumab $(n=1)$, panitumumab $(n=1)$, and vedoluzimab $(n=1)$. "Antibiotic" (superscript " $\mathrm{e}$ ") includes vancomycin $(n=52)$, unknown antibiotic $(n=14)$, cefazolin $(n=13)$, ciprofloxacin $(n=12)$, cefepime $(n=11)$, ceftriaxone $(n=11)$, levofloxacin $(n=8)$, ampicillin $(n=6)$, clindamycin $(n=6)$, sulfamethoxazole/trimethoprim $(n=$ 6), amoxicillin $(n=5)$, meropenem $(n=5)$, nafcillin $(n=4)$, ceftazidime $(n=3)$, penicillin $G(n=3)$, piperacillin/tazobactam $(n=3)$, ampicillin/ sulbactam $(n=2)$, cephalexin $(n=2)$, metronidazole $(n=2)$, azithromycin $(n=1)$, aztreonam $(n=1)$, cefotetan $(n=1)$, clarithromycin $(n=1)$, dapsone $(n=1)$, dicloxacillin $(n=1)$, imipenem $(n=1)$, minocycline $(n=1)$, moxifloxacin $(n=1)$, and tobramycin $(n=1)$. "Blood product" (superscript "f") includes red blood cells $(n=30)$, platelets $(n=28)$, immunoglobulin $(n=26)$, fresh frozen plasma $(n=15)$, unknown $(n=14)$, albumin $(n=3)$, bone marrow $(n=1)$, and stem cells $(n=1)$. "Healthcare product" (superscript "g") includes latex $(n=21)$, adhesive $(n=13)$, electrode $(n=9)$, catheter $(n=6)$, dressing $(n=2)$, filter $(n=2)$, biopatch $(n=1)$, blood pressure cuff $(n=1)$, dialyzer $(n=1)$, echocardiogram gel $(n=1)$, ink $(n=1)$, mammography equipment $(n=1)$, polysulfone dialyzer $(n=1)$, radiation $(n=1)$, strap $(n=1)$, and trach shield $(n=1)$. "Iron" (superscript " $h$ ") includes ferumoxytol $(n=16)$, feraheme $(n=9)$, iron sucrose $(n=5)$, iron dextran $(n=4)$, ferric gluconate $(n=3)$, ferritin $(n=$ 1), and unknown $(n=4)$. "Analgesic/Anesthetic" (superscript "i") includes unknown anesthetic $(n=12)$, morphine $(n=7)$, hydromorphone $(n=$ 4), ketamine $(n=2)$, oxycodone $(n=2)$, percocet $(n=2)$, acetaminophen $(n=1)$, dexmedetomidine $(n=1)$, fentanyl $(n=1)$, hydrocodone $(n=1)$, lidocaine $(n=1)$, lorazepam $(n=1)$, meperidine $(n=1)$, midazolam $(n=1)$, nalbuphine $(n=1)$, novocaine $(n=1)$, oxymorphone $(n=1)$, propofol $(n=1)$, and rocuronium $(n=1)$. "Immunosuppressant" (superscript "j") includes thymoglobulin $(n=6)$, cyclophosphamide $(n=5)$, methylprednisolone $(n=5)$, dexamethasone $(n=2)$, JTX-2011 $(n=2)$, methotrexate $(n=1)$, prednisone $(n=1)$, temsirolimus $(n=1)$, triamcinolone $(n=1)$, and unknown steroids $(n=1)$. "Food" (superscript " $k$ ") includes flavoring $(n=2)$, milk $(n=2)$, nutrition replacement $(n=$ 2), peanut $(n=2)$, applesauce $(n=1)$, coloring $(n=1)$, hot chocolate $(n=1)$, lobster $(n=1)$, mushroom $(n=1)$, parenteral nutrition $(n=1)$, raspberry yogurt $(n=1)$, walnut $(n=1)$, and unknown $(n=2)$. "Cardiovascular agent" (superscript "l") includes furosemide $(n=3)$, lisinopril $(n=3)$, captopril $(n=2)$, diltiazem $(n=2)$, epoprostenol $(n=1)$, neosynephrine $(n=1)$, unknown anti-hypertensive $(n=1)$, and vasopressin $(n=$ 1). "Anti-emetic" (superscript " $\mathrm{m}$ ") includes aprepitant $(n=3)$, fosaprepitant $(n=3)$, prochlorperazine $(n=2)$, compazine $(n=1)$, metoclopramide $(n=1)$, ondansetron $(n=1)$, and promethazine $(n=1)$. "Cleaning solution" (superscript " $n$ ") includes chlorhexidine $(n=3)$, betadine $(n=2)$, unknown cleaning agent $(n=2)$, bleach $(n=1)$, and cidex $(n=1)$. "Vitamin/Mineral" (superscript "o") includes leucovorin $(n=$

$2)$, vitamin K $(n=2)$, niacin $(n=1)$, thiamine $(n=1)$, phytonadione $(n=1)$, and iodine $(n=1)$. "Anticoagulant" (superscript "p") includes heparin $(n=1)$, aspirin $(n=3)$, prasugrel $(n=1)$, and tissue plasminogen activator $(n=1)$. "Vaccine" (superscript " $q$ ") includes pneumococcal vaccine $(n=3)$, influenza vaccine $(n=1)$, zoster vaccine $(n=1)$, and unknown vaccine $(n=1)$. "Enzyme" (superscript " $r$ ") includes asparaginase

$(n=3)$, agalsidase beta $(n=1)$, and rasburicase $(n=1)$. "Allergen" (superscript "s") includes allergen testing $(n=2)$, bee venom $(n=1)$, immunotherapy $(n=1)$, and Mantoux skin test $(n=1)$. "Antifungal" (superscript " $t$ ") includes amphotericin B $(n=4)$. "Bisphosphonate" (superscript "u") includes zoledronic acid $(n=3)$. For "Culprit" (asterisk symbol "*"), the following are not shown due to small size: abacavir $(n=1)$, efavirenz $(n=1)$, lamivudine $(n=1)$, dextrose $(n=1)$, haloperidol $(n=1)$, hydroxyethyl starch $(n=1)$, lamotrigine $(n=1)$, phenytoin $(n=$ $1)$, and probenecid $(n=1)$. 


\section{RESULTS}

Of 251,476 safety reports, 3189 (1.3\%) were confirmed allergic reactions in 2923 patients (mean age 52 years, standard deviation [SD] 17 years; $62 \%$ female). Allergic events increased over time (Fig. 1). Allergic reactions were most common in imaging $(n=1674,53 \%)$, infusion $(n=838,26 \%)$, and inpatient $(n=321,10 \%)$ settings, but also occurred in procedural $(n=121,4 \%)$ and ambulatory $(n=122,4 \%)$ settings. Most reactions resulted in temporary or minor harm $(n=2639$, $83 \%)$; fifteen $(<1 \%)$ reactions resulted in major harm or death.

Culprit allergens included diagnostic contrast agents $(n=$ $1694,53 \%)$, medications $(n=1154,36 \%)$, blood products $(n=118,4 \%)$, other healthcare products $(n=63,2 \%)$ including latex $(n=21,<1 \%)$, and foods $(n=18,<1 \%)$ (Fig. 2). Chemotherapeutics $(n=385,12 \%)$, monoclonal antibodies $(n=224,7 \%)$, and antibiotics $(n=178,5 \%)$ such as betalactams $(n=66,2 \%)$ and vancomycin $(n=52,1 \%)$ were the most common medication culprits. Culprit agents could not be identified for $164(5 \%)$ cases.

\section{DISCUSSION}

We identified 3189 allergic events from over 250,000 voluntarily reported safety events across two large AMCs. Most events occurred in imaging; over a quarter occurred in infusion settings. Over half were due to contrast media, and over one-third were due to medications, commonly chemotherapeutics, monoclonal antibodies, and antimicrobial agents; blood products, foods, and other healthcare products were also identified as culprits. Although over $80 \%$ of allergic reactions caused only temporary or minor harm, fifteen events resulted in major harm or death.

Allergic reaction treatment requires prompt identification; future allergic reaction prevention requires appropriate documentation. Unfortunately, these critical safety steps happen infrequently. ${ }^{2,7}$ Our study suggests the strongest potential for allergy safety initiatives that target radiology and infusion settings where contrast media, chemotherapeutics, and monoclonal antibodies are administered.

Because allergic events are rare and caused by a variety of culprits, healthcare systems do not explicitly track them. This machine learning model enabled allergic reaction detection from large amounts of voluntarily reported data; however, to facilitate automated downstream quality and safety improvement initiatives, the model would need to identify the culprit and reaction and demonstrate a similarly strong performance with other types of free-text clinical data (e.g., notes).

Study limitations include the voluntary nature of safety reports, including reporting culture variations between hospitals and over time. However, we reduced the impact of reporting culture by studying two large hospitals with different reporting practices. Minor reactions not warranting a safety report may have been missed. Finally, results from two AMCs in one US city may not be generalizable.
We identified and characterized allergic reactions in academic healthcare settings from a large volume of voluntarily reported safety data; we elucidate the variety of potential healthcare allergens and show that contrast media, chemotherapeutics, and biologics are the highest-risk culprits. These epidemiologic data can guide hospitals in concentrating allergy safety efforts.

Acknowledgments: The authors thank Indira S. Padubidri, MBA, Andrea Shellman, the Edward P. Lawrence Center for Quality and Safety at Massachusetts General Hospital, and the Department of Quality and Safety at Brigham and Women's Hospital for assistance in data acquisition. We thank Elizabeth Mort, MD, MPH, for review of the final manuscript and Liqing Wang, PhD, for assistance with development of the machine learning model.

Neelam A. Phadke, $M D^{1,2,3}$

Li Zhou, $\mathrm{MD}, \mathrm{Ph} \mathrm{D}^{3,4}$

Christian M. Mancini, BS ${ }^{1,5}$

Jie Yang, $P h D^{3,5}$

Paige Wickner, $M D, M P H^{3,6}$

Xiaoqing $\mathrm{Fu}, \mathrm{MS}^{1,5}$

Kimberly G. Blumenthal, MD, MSc $c^{1,3,5,7}$

${ }^{1}$ Division of Rheumatology, Allergy, and Immunology, Department of Medicine, Massachusetts General Hospital,

Boston, MA 02114, USA

${ }^{2}$ Massachusetts General Physicians Organization, Massachusetts General Hospital,

Boston, MA, USA

${ }^{3}$ Harvard Medical School,

Boston, MA, USA

${ }^{4}$ Division of General Internal Medicine, Department of Medicine, Brigham and Women's Hospital,

Boston, MA, USA

${ }^{5}$ The Mongan Institute, Massachusetts General Hospital,

Boston, MA, USA

${ }^{6}$ Division of Allergy and Clinical Immunology, Department of Medicine, Brigham and Women's Hospital,

Boston, MA, USA

${ }^{7}$ Edward P. Lawrence Center for Quality and Safety, Massachusetts General Hospital,

Boston, MA, USA

Corresponding Author: Neelam A. Phadke, MD; Division of Rheumatology, Allergy, and Immunology, Department of Medicine, Massachusetts General Hospital, Boston, MA 02114, USA (e-mail: nphadke@partners.org).

Authors' Contributions K.G.B. conceived of the project idea, secured funding, and supervised the work. J.Y. and L.Z. developed the machine learning model. C.M.M., K.G.B., and N.A.P. performed manual event review. K.G.B., N.A.P, P.W., L.Z., and X.F. developed a data analysis plan; X.F. performed data analysis. K.G.B. and N.A.P. drafted the manuscript. All authors provided critical feedback and assisted with final revision of the final manuscript.

Funding This work was supported by CRICO, the risk management foundation. 
Availability of Data The datasets generated during and/or analyzed during the current study are not publicly available as they contain peerprotected data and cannot be shared per institutional policy.

\section{Compliance with Ethical Standards:}

Conflict of Interest: The authors declare that they do not have a conflict of interest.

Ethics Approval: This study was reviewed and approved by the Partners HealthCare System Institutional Review Board.

Consent for Publication: The authors consent to publication of this manuscript.

Disclaimer: The content is solely the responsibility of the authors and does not necessarily represent the official views of CRICO.

Code Availability: The source code can be accessed at https:// github.com/jiesutd/AllergicEvent. Analysis code can be provided upon request.

\section{REFERENCES}

1. Authority PS. Medication errors associated with documented allergies. Patient Safety Advisory. 2008;5(3):75-80.
2. Barton L, Futtermenger J, Gaddi Y, et al. Simple prescribing errors and allergy documentation in medical hospital admissions in Australia and New Zealand. Clin Med (Lond). 2012;12(2):119-23.

3. Blumenthal KG, Wolfson AR, Li Y, et al. Allergic reactions captured by voluntary reporting. J Patient Saf. 2019. [Epub ahead of print].

4. Saff RR, Camargo CA Jr, Clark S, Rudders SA, Long AA, Banerji A Utility of ICD-9-CM codes for identification of allergic drug reactions. J Allergy Clin Immunol Pract. 2016;4(1):114-9.e1.

5. Saff RR, Li Y, Santhanakrishnan $\mathbf{N}$, et al. Identification of inpatient allergic drug reactions using ICD-9-CM codes. J Allergy Clin Immunol Pract. 2019;7(1):259-264.e1.

6. Yang J, Wang L, Phadke NA, Wickner PG, Mancini CM, Blumenthal KG, Zhou L. Development and Validation of a Deep Learning Model to Detect Allergic Reactions Using Safety Reports across Hospitals, 2004-2019. JAMA Netw Open. 2020 (in Press).

7. Anchor J, Settipane RA. Appropriate use of epinephrine in anaphylaxis. Am J Emerg Med. 2004;22(6):488-90.

Publisher's Note: Springer Nature remains neutral with regard to jurisdictional claims in published maps and institutional affiliations. 